\title{
How Short Is Too Short for the Interactions of a Water Potential? Exploring the Parameter Space of a Coarse-Grained Water Model Using Uncertainty Quantification
}

\author{
Liam C. Jacobson, ${ }^{\dagger}$ Robert M. Kirby, ${ }^{\ddagger}$ and Valeria Molinero*, ${ }^{\dagger}$ \\ ${ }^{\dagger}$ Department of Chemistry, The University of Utah, 315 South 1400 East, Salt Lake City, Utah 84112, United States \\ ${ }^{\ddagger}$ School of Computing, The University of Utah, 72 South Central Campus Drive, Salt Lake City, Utah 84112, United States
}

\begin{abstract}
Coarse-grained models are becoming increasingly popular due to their ability to access time and length scales that are prohibitively expensive with atomistic models. However, as a result of decreasing the degrees of freedom, coarse-grained models often have diminished accuracy, representability, and transferability compared with their finer grained counterparts. Uncertainty quantification (UQ) can help alleviate this challenge by providing an efficient and accurate method to evaluate the effect of model parameters on the properties of the system. This method is useful in finding parameter sets that fit the model to several experimental properties simultaneously. In this work we use UQ as a tool

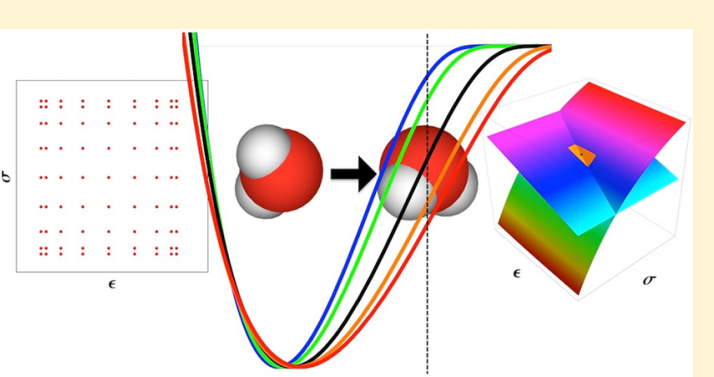
for the evaluation and optimization of a coarse-grained model. We efficiently sample the five-dimensional parameter space of the coarse-grained monatomic water $(\mathrm{mW})$ model to determine what parameter sets best reproduce experimental thermodynamic, structural and dynamical properties of water. Generalized polynomial chaos (gPC) was used to reconstruct the analytical surfaces of density, enthalpy of vaporization, radial and angular distribution functions, and diffusivity of liquid water as a function of the input parameters. With these surfaces, we evaluated the sensitivity of these properties to perturbations of the model input parameters and the accuracy and representability of the coarse-grained models. In particular, we investigated what is the optimum length scale of the water-water interactions needed to reproduce the properties of liquid water with a monatomic model with two- and three-body interactions. We found that there is an optimum cutoff length of $4.3 \AA$, barely longer than the size of the first neighbor shell in water. As cutoffs deviate from this optimum value, the ability of the model to simultaneously reproduce the structure and thermodynamics is severely diminished.
\end{abstract}

\section{INTRODUCTION}

Atomistic simulations represent a powerful set of tools to understand complex physical and chemical phenomena. Increasing computational power is giving researchers access to longer time scales and larger systems. However, methods to extend molecular simulations to further length and time scales are still needed. Coarse graining is a popular strategy to address this problem by reducing the degrees of freedom in a computational model down to a simple representation that captures essential quantities of interest (QoI) of the target system. The challenge remains to parametrize the resulting coarse-grained models so that they have adequate accuracy, representability, and transferability. Accuracy refers to faithfulness with which the coarse-grained model reproduces the value of a given experimental property at a thermodynamic state point, e.g., the density of liquid water at $298 \mathrm{~K}$ and 1 bar. Representability describes the accuracy with which the model is able to simultaneously reproduce different observables at a given state point, e.g., the density and diffusion coefficient of the liquid at $298 \mathrm{~K}$ and 1 bar. Transferability describes the ability of a model to maintain its accuracy at state points that differ from the conditions at which it was parametrized, e.g., to describe the temperature dependence of the density and the locus of the temperature of maximum density of water.
Development of coarse-grained potentials has been accomplished by several methods. In a recent perspective, Noid discussed coarse-graining in terms of "top-down" and "bottomup" approaches. ${ }^{1}$ In the "bottom-up" approach, more detailed models are used to drive the parametrization of the coarsegrained model, whereas the "top-down" approach matches macroscopic, measureable quantities directly. Force-matching, ${ }^{2,3}$ relative entropy minimization, ${ }^{4-6}$ Boltzmann inversion, ${ }^{7}$ and reverse Monte Carlo ${ }^{8}$ are coarse-graining methods that typically follow the bottom-up approach, deriving coarsegrained interaction potentials from configurations produced from atomistic simulations. Boltzmann inversion and reverse Monte Carlo have been used to develop isotropic pairwise water-water interaction potentials directly from the radial distribution functions calculated from atomistic simulations. ${ }^{9,10}$ Coarse-graining methods such as force matching $(\mathrm{FM})^{11,12}$ and relative entropy minimization $(\mathrm{REM})^{4}$ are gaining use and have been employed for the development of water models. ${ }^{2,3,13,14}$

Special Issue: James L. Skinner Festschrift

Received: February 5, 2014

Revised: March 7, 2014 
The primary limitation of REM and FM methods is that although they are grounded in well-established statistical mechanical formalisms, they are not trivial to implement in practice and do not necessarily produce properties in good agreement with the target finer-grained model from which they are derived. ${ }^{1}$ Our approach in this paper is to use the "topdown" approach to choose parameters of a coarse-grained model of water that produce output properties that minimize the residuals with experimentally determined values. A main challenge is how to efficiently sample the parameter space.

Uncertainty quantification (UQ) describes the process of quantifying uncertainties and how they are propagated in a model system of interest, such as a molecular simulation. ${ }^{15}$ The uncertainties include parametric uncertainty that results from not knowing the "true" input parameters of a model that best represent the properties of interest. UQ can be used to evaluate the impact of the parameter space on system properties and assess the sensitivity and accuracy of various parametrizations. This has traditionally been conducted using Monte Carlo (MC) sampling and its variants. In systems of large size or high dimensions, a MC simulation requires drawing a prohibitively large number of samples from probability distributions of model inputs. Numerous techniques have been proposed to reduce the computational burden associated with MC simulations, mainly by introducing more effective sampling recipes (e.g., Latin hypercube, etc.). ${ }^{16-19}$ Although these developments significantly improve the tractability of MC approaches, there is still a need for more efficient approaches.

Among the methods developed as alternatives to $\mathrm{MC}$ simulation, the generalized polynomial chaos (gPC) methodology has been proposed as a more efficient way to address many UQ questions. ${ }^{20-22}$ It consists of a spectral approximation of random variables in the stochastic domain, where random variables are expressed as series expansions involving orthogonal polynomials. The quantities of interest are also represented using a truncated expansion based on orthogonal polynomials, effectively constituting a finite-dimensional approximation of the infinite-dimensional stochastic space. Because polynomial basis functions are used, random QoI can be effectively interpolated on the basis of the tensor product of a few samples drawn from each input random variable. This leads to significant computational savings. Given the resulting functional forms for QoI, one can then readily use the analytical expressions derived for quantities such as statistical moments and sensitivity indices. ${ }^{23}$

Sampling based on the tensor product of one-dimensional sample points is only tractable for problems of low dimensions, i.e., problems having at most around five random parameters. That is because the number of required samples to reliably compute the QoI grows exponentially with the dimension, unless more efficient sampling techniques are exploited. To this end, high-order stochastic collocation approaches have been introduced. ${ }^{24-26}$ It is still an open area of research to further enhance the convergence behavior of the stochastic collocation method to high dimensional problems where a large number of random parameters, e.g., on the order of 100, are involved, although there are some proposed methodologies within the literature. Another challenge associated with the classical form of the gPC expansion is their limited applicability to smooth behaviors. To expand its applicability, in addition to original smooth polynomials, e.g., Hermite or Legendre polynomials, chaos expansions based on generalized basis functions have been developed. Examples of this generalization include wavelet basis functions, ${ }^{27,28}$ piecewise polynomial functions, ${ }^{29}$ and multielement gPC expansions. ${ }^{30}$

UQ with gPC is a powerful tool to guide and refine force field development and evaluate the representability of atomistic and coarse-grained models. Rizzi et al. have used UQ to evaluate the impact of parameter space on the density, enthalpy, and diffusivity of the TIP4P water model ${ }^{31,32}$ as well as to investigate the interplay of parametric uncertainty and intrinsic noise on the flux of ions through silica nanopores. ${ }^{33,34}$ They showed that the thermal noise present in molecular dynamics simulations is small in comparison to the parametric uncertainty of the model and that, once the fluctuations are averaged over, there is a smooth dependence on the input parameters. These findings support the use of a polynomial chaos expansion to deterministically represent the macroscopic observables as a function of model parameters. This is the approach that we follow in this work. Rizzi et al. presented a proof of concept use of UQ to determine the "true" input parameters for the TIP4P water model from the results of simulations from those very same input parameters as a way to validate the use of UQ to parametrize a model. Here we use UQ to determine parameters that reproduce multiple experimental properties of interest simultaneously in a coarsegrained model of water, and to assess the effect of the length of the interaction potential on the accuracy and representability of the water models.

Several paradigms for coarse-grained water models have been proposed in the literature. The standard rigid atomistic water models with electrostatic interactions remain the most popular class of water models and can be considered coarse-grained representations of more detailed flexible or quantum mechanical models of water. Models that keep the full atomistic representation of water but reduce the length scale of the effective interactions ${ }^{35,36}$ represent the next level of coarsegraining. Further coarsening can be attained by representing the water molecule as a single particle. ${ }^{37}$ Within this paradigm there are two main classes of potentials: those that are isotropic, ${ }^{2,38-41}$ and those that introduce anisotropic terms to represent the directionality of hydrogen bonding interactions between water molecules. 33,42,43 Isotropic coarse-grained water models have been mostly parametrized from atomistic simulations. Izvekov and Voth used force-matching to create a multiscale coarse-grained representation of water as a single site that was able to reproduce the radial distribution function and density of liquid water. ${ }^{2}$ Johnson et al. derived coarse-grained effective pair potentials from TIP4P-Ew and demonstrated that isotropic pair potentials are unable to simultaneously reproduce the structure and equilibrium thermodynamic properties. 39,40 Chaimovich and Shell developed spherically symmetric coarsegrained potentials of water at various state points using relative entropy minimization. ${ }^{38}$ A limitation of monatomic isotropic water models is their lack of transferability to state points beyond those used in their parametrization. ${ }^{38}$ Among the single particle models with anisotropic interactions, there are models that explicitly include three-body interactions, such as the monatomic water $(\mathrm{mW})$ model $^{42}$ and the models of Larini et al., ${ }^{3,13}$ and models such as the 3D Mercedez Benz model, ${ }^{43}$ which consider pair interactions between particles that have hydrogen bonding "arms" at fixed orientations. Models that represent multiple water molecules as a single particle, with or without anisotropic interactions, represent a further notch up in coarsening. ${ }^{44-46}$ 
The first aim of this work is to use UQ for the evaluation and optimization of parameters for the monatomic water $(\mathrm{mW})$ model using various metrics to account for the representability and transferability of the resulting potentials. $\mathrm{mW}$ represents each water molecule as a single site that interacts with its neighbors through two- and three-body interactions with the form of the Stillinger-Weber potential. ${ }^{42}$ The three-body terms favor the tetrahedral configurations that are characteristic of water. This results in "hydrogen-bonded" configurations without the computational cost of long-range electrostatics or the need to integrate the very fast rotational dynamics of the water molecules in the liquid. The incorporation in $\mathrm{mW}$ of three-body potentials that mimic hydrogen bonds resulted in significant increases in representability and transferability compared with the cases of isotropic monatomic potentials and opened the possibility of using coarse-grained models to study hydrophobic interactions, liquid anomalies, and crystallization of water. ${ }^{42,47-53}$

The original "top-down" parametrization of $\mathrm{mW}$ privileged the reproduction of the experimental melting temperature $T_{\mathrm{m}}$ of ice I at $1 \mathrm{bar}$, and the enthalpy of vaporization and density of liquid water at $1 \mathrm{bar}$ and $298 \mathrm{~K}$. In this work, in addition to evaluating the thermodynamic properties that were used as metrics in the original $\mathrm{mW}$ model, we consider structural properties (radial and angular distribution functions) as benchmarks for the parametrization of the coarse-grained water model. We find that optimizing the density and enthalpy of vaporization with the radial distribution function instead of the temperature of melting results on a coarse-grained water model that is very close to $\mathrm{mW}$ and with similar overall agreement in reproducing water properties.

The second aim of this work is to investigate what is the effect of decreasing the length scale of the interactions of the water model on its accuracy and representability. A strong motivation to minimize the interaction length of coarse-grained water models is to further decrease their computational costs. Bernal and Fowler recognized over 80 years ago that most of the intermolecular potential energy of water in condensed phases is accounted for by interactions within the first neighbor shell. ${ }^{54}$ They estimated that electrostatic interactions beyond the first neighbor shell contributed only about $10 \%$ of the enthalpy of sublimation of ice. Coarse-grained models of water exploit the effective short range of water-water interactions for performance gains by using a relatively short interaction length compared to fully atomistic models based on long-range electrostatics. Weeks and co-workers developed fully atomistic "short water" SPC/E models replacing the long-range coulomb interaction of the atomic sites by short-range potentials, corresponding to the screened electrostatic potential that represents a point charge surrounded by neutralizing Gaussian charge distribution of width $\sigma_{\mathrm{C}}=4.5 \AA$ and either keeping the full Lennard-Jones interaction $\left(\sigma_{\mathrm{LJ}}=3.16 \AA\right)$ or replacing it by a purely repulsive WCA potential. ${ }^{35,55}$ They showed that both types of short water models were able to model a hydrogen bond network, but the attractive Lennard-Jones potential was needed to observe the density anomaly that is a hallmark of liquid water. Interactions in the monatomic water model $\mathrm{mW}$ have a cutoff length of $4.31 \AA$, extending just beyond the first neighbor shell ( $3.5 \AA)$. The short-ranged nature of the potential along with the lack of hydrogen atoms results in simulations over 100 times computationally more efficient than for atomistic models with electrostatics. Following the same modeling paradigm of $\mathrm{mW}$, Larini et al. used force matching to derive water potentials from SPC/E water using the threebody term of the Stillinger-Weber potential and spline functions for the pairwise interactions. ${ }^{3,13}$ Those models have even shorter water-water interaction lengths than $\mathrm{mW}, 3.7-$ $3.9 \AA$, and successfully reproduce the radial distribution function (rdf) and angular distribution function (adf) of the atomistic SPC/E model, but they severely underestimate $\Delta H_{\text {vap}}$, which was about $60 \%$ lower than the target value, ${ }^{3}$ and does not display a density maximum. ${ }^{14}$ Water models similar in quality were derived using relative entropy minimization. ${ }^{14}$ The striking difference in performance and properties between $\mathrm{mW}$ and the slightly shorter monatomic anisotropic models derived with force matching and relative entropy minimization poses the questions of what is the role of the length of the interaction potential on the representability of the coarse-grained water models. How short can a water model be? We find that for models that follow the $\mathrm{mW}$ paradigm there is a very narrow region of interaction length that allows for a good reproduction of the structure and energetics of water. That optimum length scale is $4.29 \AA$, only slightly larger than the first neighbor shell in liquid water.

\section{MODEL AND SIMULATION METHODS}

A. $\mathrm{mW}$ Water Model. In this work we explore the parameter space of the $\mathrm{mW}$ water model, ${ }^{42}$ which has the form of the Stillinger-Weber (SW) potential originally developed for silicon: ${ }^{56}$

$$
\begin{aligned}
& \sum_{i} \sum_{j>i} \phi_{2}\left(r_{i j}\right)+\sum_{i} \sum_{j \neq i} \sum_{k>j} \phi_{3}\left(r_{i j}, r_{i k}, \theta_{i j k}\right) \\
& \phi_{2}\left(r_{i j}\right)=A \varepsilon\left[B\left(\frac{\sigma}{r_{i j}}\right)^{4}-1\right] \exp \left(\frac{\sigma}{r_{i j}-a \sigma}\right) \\
& \phi_{3}\left(r_{i j}, r_{j k}, \theta_{i j k}\right) \\
& \quad=\lambda \varepsilon\left[\cos \theta_{i j k}-\cos \theta_{0}\right]^{2} \exp \left(\frac{\gamma \sigma}{r_{i j}-a \sigma}\right) \exp \left(\frac{\gamma \sigma}{r_{i k}-a \sigma}\right)
\end{aligned}
$$

The constants $A$ and $B$ define the form of the potential and are kept fixed to the original SW values $A=7.049556277$, and $B=$ 0.6022245584 . The parameter $\theta_{0}$ was maintained at the preferred tetrahedral angle, $109.47^{\circ}$, for all simulations. The parameter space is defined by the five parameters $\varepsilon, \sigma, a, \lambda$, and $\gamma$. Section III details the strategy and procedure for sampling the parameter space around the values of $\mathrm{mW}: \varepsilon=6.189 \mathrm{kcal} /$ mol, $\sigma=2.3925 \AA$, $a=1.8, \lambda=23.15, \gamma=1.2$.

B. Simulation Details. Molecular dynamics simulations of systems modeled with more than a thousand distinct set of parameters were performed using LAMMPS. ${ }^{57}$ Each simulation system consisted of a periodic cell with 4096 water molecules and was performed in the isothermal, isobaric ensemble at a temperature of $298 \mathrm{~K}$ and pressure $1 \mathrm{~atm}$ using the NoseHoover thermostat and barostat with damping parameters of 5 and $25 \mathrm{ps}$, respectively. Each simulation had a time step of $10 \mathrm{fs}$ and was performed for $10 \mathrm{~ns}$, with properties averaged over the last 9 ns. Additional simulations employed to compute melting temperatures and the surface tensions and widths of the liquidvapor interface are described in next section.

C. Calculation of Properties. We calculated the enthalpy of vaporization, density, diffusion coefficient, radial distribution 
function and angular distribution function for each simulation and compared them with experimental values. The enthalpy of vaporization $\Delta H_{\text {vap }}$ of water was calculated as $\Delta H_{\text {vap }}=2.5 R T-$ $\langle E+p V\rangle$, where $R$ is the gas constant, $E$ is the total internal molar energy, $p$ is the pressure, and $V$ is the molar volume. The 2.5RT term results because we assume the gas phase has the same enthalpy as an ideal gas with zero internal energy.

Density $\rho$ was calculated as $\rho=N M /\left(N_{\mathrm{A}}\langle V\rangle\right)$, where $N$ is the number of water molecules and $M$ is the molecular mass of water $18.015 \mathrm{~g} / \mathrm{mol}$. Values were compared to the experimental density of liquid water of $0.997 \mathrm{~g} / \mathrm{mL}$ at $298 \mathrm{~K}$ and $1 \mathrm{~atm}$.

The self-diffusion coefficient $D$ was determined using Einstein's relation

$$
D=\lim _{t \rightarrow \infty} \frac{1}{6 t}\left\langle|r(t)-r(0)|^{2}\right\rangle
$$

where $t$ is the time of the simulation and $\left\langle|r(t)-r(0)|^{2}\right\rangle$ is the root-mean-square displacement.

The radial distribution function, or rdf, is a measure of the liquid structure and was calculated as

$$
g_{i j}(r)=\frac{V}{N^{2}}\left\langle\sum_{i} \sum_{j \neq i} \delta\left(r-r_{i j}\right)\right\rangle
$$

The rdf was compared to experimental determinations by Skinner et al. ${ }^{58}$ The residual of the $\mathrm{rdf}$ with respect to the experimental measurement is calculated as the square of the difference between the simulation $\mathrm{rdf}$ and the experimental $\mathrm{rdf}$ as

$$
\operatorname{res}_{\text {rdf }}=\sum_{i=1}^{N}\left|\operatorname{rdf}_{\text {exp }}\left(r_{i}\right)-\operatorname{rdf}_{\text {sim }}\left(r_{i}\right)\right|^{2} \Delta r
$$

where $\operatorname{rdf}_{\text {exp }}$ and $\mathrm{rdf}_{\text {sim }}$ are the values of the radial distribution functions from experiment and simulation, respectively. Here, $N=r_{\mathrm{c}} / \Delta r$, where $r_{\mathrm{c}}$ is the cutoff distance and $\Delta r$ is the distance between points sampled from the rdf. In this work, we used a cutoff $r_{\mathrm{c}}=10 \AA$ and sampled using $\Delta r=0.06 \AA$.

The angular distribution function, or adf, was calculated as

$$
P(\theta)=\frac{1}{N n_{\theta}}\left\langle\sum_{k=1}^{N} \sum_{i=1}^{n_{c}} \sum_{j \neq 1}^{n_{c}-1} \delta\left(\theta-\theta_{i k j}\right)\right\rangle
$$

where $n_{\theta}$ is the number of angles, $n_{\mathrm{c}}$ is the number of neighbors, and $N$ is the number of particles. The calculated adf was compared to the adf determined from the experimental results of Strässle et al. ${ }^{59}$ The residual of the adf with respect to the experimental measurement is calculated as the square of the difference between the simulation adf and the experimental adf.

$$
\operatorname{res}_{\text {adf }}=\sum_{i=1}^{180} \operatorname{ladf}_{\exp }\left(\theta_{i}\right)-\left.\operatorname{adf}_{\text {sim }}\left(\theta_{i}\right)\right|^{2}
$$

where $\operatorname{adf}_{\text {exp }}$ and $\operatorname{adf}_{\text {sim }}$ are the values of the angular distribution functions from experiment and simulation, respectively. In this work, we sampled the adf every 1 degree between 1 and 180 .

The liquid-vapor surface tension $\gamma_{\mathrm{lv}}$ for each set of parameters was determined from simulations of a liquid slab as described in ref 60 from the pressure tensor components normal $\left(p_{\mathrm{n}}\right)$ and tangential $\left(p_{\mathrm{t}}\right)$ to the slab surface averaged over equilibrium trajectories:

$$
\gamma_{\mathrm{lv}}=\frac{L_{z}}{2}\left[\left\langle p_{\mathrm{n}}\right\rangle-\left\langle p_{\mathrm{t}}\right\rangle\right]
$$

where $L_{\mathrm{z}}$ is the length of the simulation cell in the direction perpendicular to the surface.

The width of the liquid-vapor interface $t_{90-10}$ is defined as the width over which the density of liquid water decays from $90 \%$ to $10 \%$ of the bulk density, computed from density profiles in the direction perpendicular to the interface and averaged over simulations of the water slab.

The temperature of maximum density $T_{\mathrm{MD}}$ was calculated by performing an isobaric cooling of the liquid from 320 to $205 \mathrm{~K}$ at a cooling rate of $0.5 \mathrm{~K} / \mathrm{ns}$. A running average of densities was taken as a function of temperature and the temperature with the maximum density was identified. We also determined $T_{M D}$ using the interpolation methods described in section III.

The melting temperature $T_{\mathrm{m}}$ of hexagonal ice $I_{\mathrm{h}}$ was determined using the method of direct coexistence in the $\mathrm{NPH}$ ensemble as described by Wang et al. ${ }^{61}$ The simulation consisted of 9216 atoms, half $I_{\mathrm{h}}$ lattice and half liquid. The time step for the melting temperature simulations was 5 fs.

\section{INTERPOLATION METHODS}

To construct the response surfaces of the output properties, we use a generalized polynomial chaos collocation approach. For simplicity, we use tensor products of one-dimensional interpolatory functions to build our multidimensional surfaces. Chebyshev nodes reduce the error of the interpolation compared to equidistant nodes. They are nested and geometrically hierarchical for odd numbers of points, so points can be reused in subsequent interpolations, requiring fewer simulations. In addition, with appropriate weights, Chebyshev points can be used to form quadrature rules (i.e., ClenshawCurtis quadrature), hence allowing us to compute statistical moments (e.g., expectation, etc.). Simulations were run using parameters defined by Chebyshev nodes in each dimension that are generated over the interval $[a, b]$ using eq 8 ,

$$
x_{i}=a+\frac{b-a}{2}\left(1+\cos \left(\frac{(2 i+1) \pi}{(n+1) 2}\right)\right) \quad i=0,1, \ldots, n
$$

The nodes are located at roots of Chebyshev polynomials, and each node represents a simulation using that parameter.

Lagrange interpolation was performed using polynomials generated from eqs 9 and 10,

$$
\begin{aligned}
& h_{k}(x)=\prod_{\substack{i=1 \\
i \neq k}}^{N} \frac{\left(x-x_{i}\right)}{\left(x_{k}-x_{i}\right)} \\
& p(x)=\sum_{k=1}^{N} f_{k} h_{k}(x)
\end{aligned}
$$

where $x_{k}$ represent the points in parameter space, and $f_{k}$ represent the measured values of the observable from the simulation using that particular value parameter. The resulting polynomial $p(x)$ is a response function that can be used to determine the value of the observable from a molecular dynamics simulation as a function of the parameter $x$. Figure 1 shows Chebyshev nodes generated from eq 8 for $n=0,1$, and 3 as well as response curves $p(x)$ constructed using eqs 9 and 10 representing the density of liquid water as a function of temperature.

This method can be extended to higher dimensions using a tensor product approach. The following equation is used to 


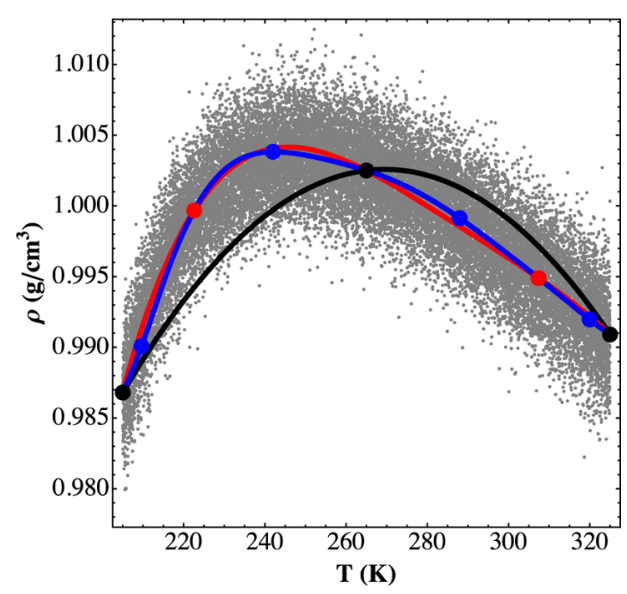

Figure 1. Lagrange interpolation for the response function describing density of liquid water as a function of temperature using Chebyshev points defined in eq 8 ( $a=205$ and $b=325)$ for $n=0$ (black points), 1 (red and black points), and 3 (blue, red and black points). The response functions $p(x)$ are constructed using eqs 9 and 10, where $x_{k}$ represent the temperatures and $f_{k}$ represent the density from a simulation using that particular temperature. The accuracy of the response function increases with the number of points used to construct the interpolating polynomial $(3,5$, and 9 points in the black, red, and blue curves, respectively). The temperature of maximum density $T_{\mathrm{MD}}$ for the $\mathrm{mW}$ water model is determined from a slow rate cooling simulation of liquid water from 325 to $205 \mathrm{~K}$ (gray points). The nine short simulations used to construct the response function require only a fraction of the computational resources compared to the isobaric quench.

reconstruct the response surfaces on the basis of the parameters $\sigma$ and $\varepsilon$

$$
p(\sigma, \varepsilon)=\sum_{i=1}^{N} \sum_{i=1}^{N} f_{i j} h_{i}(\sigma) h_{j}(\varepsilon)
$$

This method can be further extended to additional dimensions as

$$
p\left(x_{1}, x_{2}, \ldots, x_{n}\right)=\sum_{i=1}^{N} \sum_{j=1}^{N} \ldots \sum_{m=1}^{N} f_{i j \ldots m} h_{i}\left(x_{1}\right) h_{j}\left(x_{2}\right) \ldots h_{m}\left(x_{n}\right)
$$

The $\mathrm{mW}$ model has five parameters, so building a response surface for all parameters simultaneously would be prohibitively expensive, requiring at least $n^{5}$ simulations, with $n$ being the number of points in each dimension required to accurately construct the response (i.e., physical property) surface. To overcome this challenge to the parametrization, we prioritize specific parameters $(\varepsilon, \sigma, a, \lambda$, and $\gamma)$ using a physical basis. For each parameter, we sampled 3,5 , or 9 points in a range $20 \%$ above and below the parameter values for the standard $\mathrm{mW}$ water model. For parameters $\varepsilon, \sigma$, and $\lambda$, we constructed a $9 \times 9$ $\times 9$ tensor product grid consisting of 729 simulations. For parameters $a$ and $\gamma$, we constructed a $9 \times 9$ tensor product grid consisting of 81 simulations. For parameters $a, \sigma$, and $\lambda$, we constructed a $5 \times 5 \times 5$ tensor product grid consisting of 125 simulations. As noted earlier, a tensor product construction of our response surfaces suffers from the "curse of dimensionality"; this approach can become intractable as the number of parameters (and hence dimension) increases. In an attempt to overcome these challenges, sparse grid and adaptive high-order stochastic collocation approaches have been introduced. ${ }^{24-26}$
The hierarchical nature of the Lagrange interpolation allows for efficient sampling. The precision of the interpolation increases with the number of points, but the number of points increases exponentially as we go from one level of interpolation to the next. Hence, the nested nature of this method benefits from an iterative approach. To address some of the challenges posed by the high dimensionality of parameter space of the coarse-grained $\mathrm{mW}$ potential, we evaluated the level of precision needed to reconstruct the response surfaces for the parameter space of the $\mathrm{mW}$ water model. Each response surface that is used as a proxy for the parameter search requires a different level of precision. Surfaces that are smooth and vary linearly with parameter perturbations gain little with additional resolution. For instance, changing the density of points in the tensor grid for $\Delta H_{\text {vap }}$ from $3 \times 3$ to $5 \times 5$ yields a difference of only $0.4 \%$, and increasing the grid density from $5 \times 5$ to $9 \times 9$ yields a difference of $0.3 \%$. Clearly, the additional computation of the higher precision surface is not worth the additional resources. However, for a response surface that is rough, i.e., more sensitive to input parameters, such as the residual of the rdf (eq 4) as a function of $\varepsilon$ and $\sigma$ for a single value of $\lambda$, as shown in Figure 2, going from $3 \times 3$ to $5 \times 5$ yields a difference of $37 \%$, and going from $5 \times 5$ to $9 \times 9$ yields a difference of $5 \%$. The accuracy of this surface certainly benefits from the additional simulations. The accuracy of the interpolating polynomial also depends on the size of the interpolation interval. A smaller interval may require fewer points to accurately reproduce the response surface, but too small an interval could potentially leave out a region of interest. This underscores the utility of an iterative approach using Chebyshev points. Initial simulations can cover a wide range of parameter space, and additional resolution can be focused on narrower regions of interest while some of the previous interpolation points are reused. These methods can be extended for the parametrization of new force fields by limiting the amount of computational resources expended to only what is necessary to understand the impact of the parameter space.

In addition to understanding the effect of changing the model parameters of the interaction potential, these methods can be used to determine the impact of the state space on the output properties of the simulation. UQ can be used evaluate the transferability of a model over a wide variety of state points. The higher sampling efficiency of Lagrange interpolation can be used to construct response functions such as the dependence of density on temperature with fewer computational resources as shown in Figure 1. The determination of $T_{M D}$ from an isobaric quench that is slow enough to relax to a local equilibrium at each temperature $(0.5 \mathrm{~K} / \mathrm{ns})$ required more than 28 times as much computational resources as constructing the response surface from 9 simulations with the state points defined by Chebyshev nodes.

\section{SENSITIVITY OF THE PROPERTIES OF THE WATER MODEL TO INPUT PARAMETERS}

We investigated the effect of changing input model parameters on the output properties of coarse-grained models of the family of $\mathrm{mW}$ water. Water models with a common functional form, but different parametrizations designed to meet different benchmarks have been previously established (e.g., the TIP4P family of four-point water models or the family of three-point models encompassing SPC, SPC/E and TIP3P). TIP4P, for example, was originally parametrized to reproduce the enthalpy of vaporization and density of liquid water at ambient 

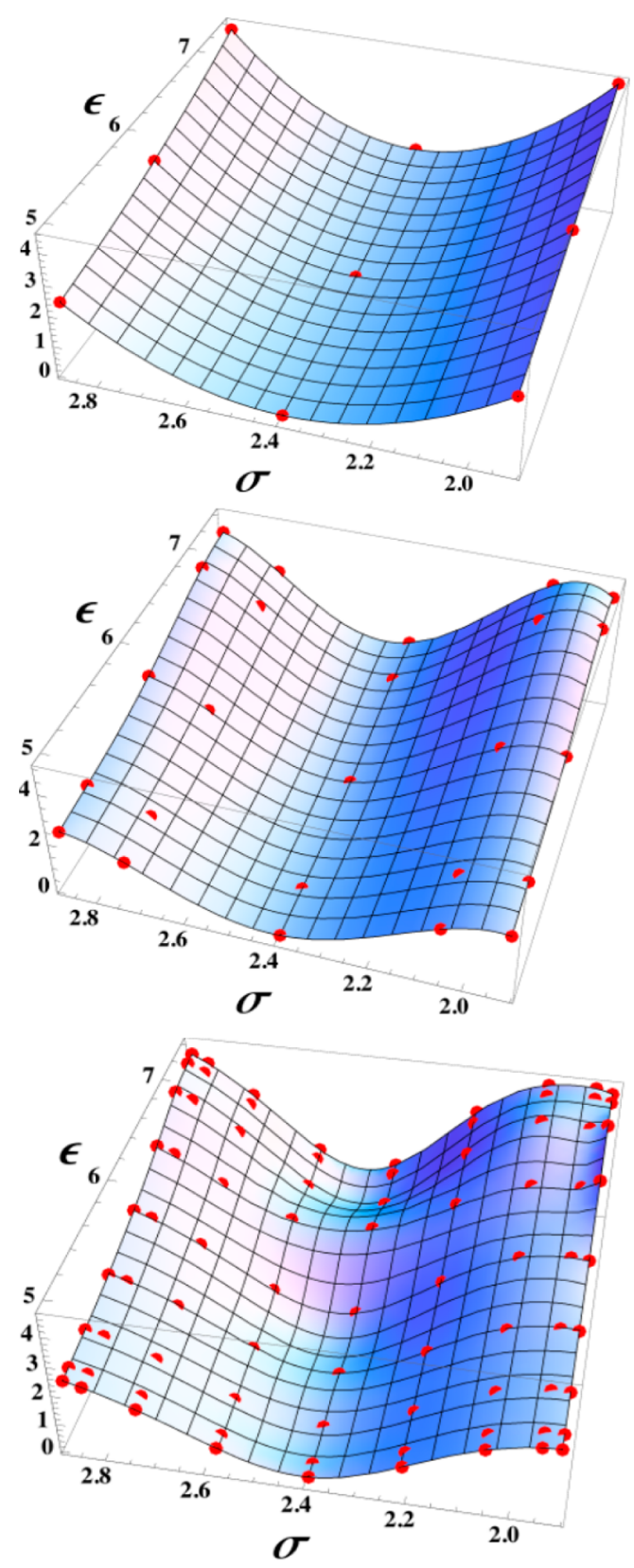

Figure 2. Response surface representing the residual of the $\mathrm{rdf}$ as a function of $\varepsilon$ and $\sigma$ (all other parameters as in $\mathrm{mW}$ water). The same response surface is shown using $3 \times 3$ (upper), $5 \times 5$ (middle), and 9 $\times 9$ (lower) tensor grids. Increasing the density of Chebyshev points (red dots) increases the accuracy of the response function but comes at the cost of more simulations.

conditions. ${ }^{62}$ It was later refined to match the temperature of maximum density and work with Ewald sums, resulting in TIP4P-Ew. ${ }^{63}$ It was further reparametrized as TIP4P-Ice to reproduce the $T_{\mathrm{m}}$ of ice, but sacrificing the accuracy in the enthalpy of vaporization for better agreement with global properties. $^{64}$ Vega and Abascal provided a comprehensive summary of properties of rigid nonpolarizable atomistic models of water and demonstrated that trade off in the accuracy in certain properties results in dramatic changes in the global representability of the models. ${ }^{62}$

Due to the effect of averaging over degrees of freedom, coarse-grained models are not capable of reproducing all properties of interest of the finer resolution model (or the experimental system) simultaneously. Therefore, when a coarse-grained model is parametrized, some properties must be prioritized and it must be ensured that the model derived has adequate fidelity for those properties. The parametrization of the $\mathrm{mW}$ model privileged $T_{\mathrm{m}}$ of ice, and the enthalpy of vaporization and density of liquid water at $298 \mathrm{~K}$. The development of $\mathrm{mW}$ followed a noniterative procedure to determine the value of three parameters $(\varepsilon, \sigma$, and $\lambda)$ from these three experimental properties. ${ }^{42}$ First, $T_{\mathrm{m}}$ was calculated in reduced units as a function of $\lambda$ and $\varepsilon$ was scaled such that for each value of $\lambda T_{\mathrm{m}}$ was $273.15 \mathrm{~K}$. Then, the enthalpies of melting, vaporization, and sublimation were determined as a function of $\lambda$, and the $\lambda$ that resulted in enthalpies that most closely matched experiment was chosen. Finally, $\sigma$ was scaled so that the density of the simulation matched the experimental density. In this work we take a different approach, we construct surfaces for properties describing the thermodynamics, structure, and diffusivity of liquid water as a function of the $\varepsilon, \sigma, a, \lambda, \gamma$ parameters of the Stillinger-Weber potential to explore alternate parametrizations of the coarse-grained water model using as benchmarks experimental properties that account not only for the energetics but also for the structure of liquid water.

The properties used in the parametrization of this work describe the thermodynamics, structure, and diffusivity of liquid water. Two properties were considered as metrics of water structure: the radial distribution function ( $\mathrm{rdf}$ ) of liquid water determined from recent $\mathrm{X}$-ray diffraction measurements, which improve upon previous determinations and reduce the associated truncation error, ${ }^{58}$ and the angular distribution function (adf) for the closest eight neighbors. ${ }^{59}$ The density $\rho$ of liquid water is a thermodynamic property that provides an additional measure of the faithfulness of the model in reproducing the structure and characteristic intermolecular distances in liquid water. The enthalpy of vaporization $\Delta H_{\text {vap }}$ serves as our benchmark for the energetics of liquid water. Other energetic observables such as the enthalpy of melting were computed and could be used as well but, as we discuss below, each of these various properties results in a different set of optimum parameters, because not all these properties can be simultaneously reproduced by the model. The dynamics of water was measured through the self-diffusion coefficient $D$.

Because coarse-grained models average out some of the degrees of freedom of a fully atomistic system, not all the entropy of water is accounted for in the monatomic water model. Coarse-grained models also have a smoother potential energy surface with lower kinetic barriers. These factors, and the known scaling between entropy and diffusivity, ${ }^{47,65-68}$ preclude the ability of the coarse-grained model to simultaneously reproduce the energetics and dynamics of water. Therefore, we focus on maximizing the accuracy of properties that describe the energetics and the structure of liquid water.

To optimize the model, we find the parameters that minimize the difference between the calculated output properties and those measured in experiments. Ideally, the properties used as benchmarks for the parametrization must be calculable from a simulation at a single state point. Such is the case for density, enthalpy of vaporization, diffusion coefficient, rdf, and adf. It is possible to use a measurement that requires multiple simulations for each individual parameter set, but it adds to the computational complexity and expense of the parametrization, which increases as a power function with each parameter. Properties such as the melting temperature $T_{\mathrm{m}}$ 
melting enthalpy $\Delta H_{\mathrm{m}}$, melting entropy $\Delta S_{\mathrm{m}}$, surface tension $\gamma_{\mathrm{lv}}$ and width $t_{90-10}$, and temperature of maximum density $T_{\mathrm{MD}}$ are less amenable to this approach and were only calculated for individual parameter sets. Sampling the full parameter space of the five parameters of $\mathrm{mW}$ model $(\varepsilon, \sigma, a, \lambda, \gamma)$ would require at least $9^{5}$ (about 60000 ) simulations. Here we focus on the three parameter spaces defined by smaller sets, $(\varepsilon, \sigma, \lambda),(a, \gamma)$, and $(a, \sigma, \lambda)$, which are sampled with approximately 1000 simulations.

To illustrate how we use response surfaces to choose parameters, we start with the parameter set $(\varepsilon, \sigma, \lambda)$, which has been partially explored for the Stillinger-Weber potentials in the literature. ${ }^{42,47,48,68,69}$ We constructed response surfaces using the interpolating polynomials described in eqs 9 and 12 to determine the output properties of $\Delta H_{\text {vap }}$ and $\rho$ as a function of the parameters. The interpolating polynomials were generated from the basis set of observations from the 729 simulations that spanned the parameter space $(\varepsilon, \sigma, \lambda)$. All other parameters were fixed to their values in the standard $\mathrm{mW}$ potential. Figure 3 shows the response surfaces for $\Delta H_{\text {vap }}$ and

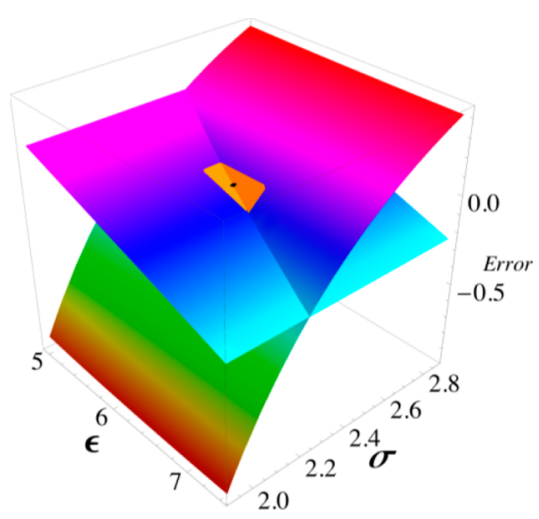

Figure 3. Response surfaces describing the relative error of the enthalpy of vaporization and density of liquid water with respect to experimental values as a function of $\varepsilon$ and $\sigma$. The surface for the enthalpy of vaporization varies as a function of $\varepsilon$, and the response surface for the density is dependent on $\sigma$. There is only one unique point at which the two surfaces simultaneously match the experimental results. The black dot in the center marks the point in parameter space that matches both the experimental density and $\Delta H_{\text {vap }}(\varepsilon=6.121$ $\mathrm{kcal} / \mathrm{mol}$ and $\sigma=2.3916 \AA$; all other values are the same as in the $\mathrm{mW}$ model). The orange box shows the region in parameter space that is within 5\% of the experimental values for both properties. As discussed in section III, the response surfaces for the enthalpy and density were accurately reproduced with the smallest set of points $(3 \times 3$ tensor grid).

density as a function of $\varepsilon$ and $\sigma$ while $\lambda$ is maintained at 23.15, the same as in the original $\mathrm{mW}$ model. The optimal parameters were determined by finding the parameter set on the response surface that simultaneously reproduces the experimental density and enthalpy of vaporization. Because both surfaces vary monotonically with $\varepsilon$ and $\sigma$, there is only one point in the parameter space that can simultaneously fulfill these criteria. For the parameter space $(\varepsilon, \sigma)$ maintaining $\lambda=23.15$, we determined that the parameters $\varepsilon=6.121 \mathrm{kcal} / \mathrm{mol}$ and $\sigma=$ $2.3916 \AA$ simultaneously reproduced the experimental values for the enthalpy of vaporization and density of liquid water. Not surprisingly these values are close to the values of the standard $\mathrm{mW}$ model $(\varepsilon=6.189 \mathrm{kcal} / \mathrm{mol}$ and $\sigma=2.3925 \AA)$ that did not use the structure as part of the parametrization. Next we will introduce the structure as an additional metric in the parametrization.

The ability of a model potential to reproduce the structure of liquid water was measured by the residuals between the rdf and adf in the simulations and experiment $\left(\mathrm{rdf}_{\text {res }}\right.$, eq 4 and $\mathrm{adf}_{\mathrm{res}}$, eq $6)$. Figure 3 illustrates that the response surfaces for $\Delta H_{\text {vap }}$ and $\rho$ are dependent on $\varepsilon$ and $\sigma$, respectively. Unlike the case for $\Delta H_{\text {vap }}$ and $\rho$, no parameter set was able to exactly reproduce the experimental rdf. The response surfaces for $\operatorname{rdf}_{\text {res }}$ and adf for the parameter space $(\varepsilon, \sigma)$ with all other parameters as in $\mathrm{mW}$ water are shown in Figure 4. It is evident that the ability to

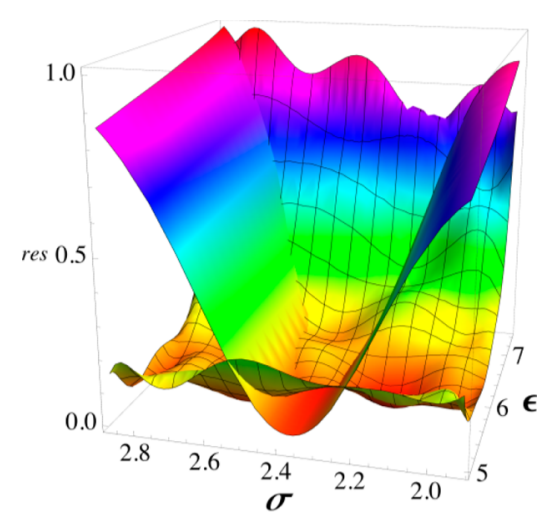

Figure 4. Response surfaces of the residuals for the radial distribution function $\operatorname{rdf}_{\mathrm{res}}$ (unmeshed surface) and the angular distribution function $\operatorname{adf}_{\text {res }}$ (meshed surface) with respect to experiment as a function of $\varepsilon$ and $\sigma$ (all other parameters the same as in the $\mathrm{mW}$ model). The response surface for $\mathrm{rdf}_{\text {res }}$ is more sensitive to the input parameters than that of adf res. To aid in the comparison, the adf $\mathrm{res}_{\text {res }}$ and $\mathrm{rdf}_{\text {res }}$ have been scaled so that both surfaces have a maximum value of 1. As discussed in section III, the response surfaces for the $\mathrm{rdf}_{\mathrm{res}}$ and $\mathrm{adf}_{\text {res }}$ require a higher number of interpolation points than the enthalpy of vaporization and density; 81 simulations $(9 \times 9$ tensor grid) were used to interpolate the surfaces shown here.

reproduce the experimental $\mathrm{rdf}$ is more selective to the choice of parameters than the adf. This may be because our determination of the adf uses the closest eight neighbors. Computing the adf using the nearest four neighbors or using a tetrahedral order parameter considering only first shell neighbors may yield a better method to quantify the tetrahedrality of the liquid, but we lack the corresponding benchmarks derived from experiment. Due to the higher selectivity for parameters of the rdf compared to the adf, we used the rdf as the primary metric for the structure of liquid water.

As we described above, for each value of $\lambda$, there is a combination of $\varepsilon$ and $\sigma$ that simultaneously reproduces $\Delta H_{\text {vap }}$ and $\rho$. Including $\lambda$ as a degree of freedom in the optimization, we used the 729 simulations that spanned the parameter space $(\varepsilon, \sigma, \lambda)$ to construct interpolating functions that allowed us to determine the set of parameters $(\varepsilon, \sigma)$ that reproduce $\Delta H_{\text {vap }}$ and $\rho$ as a function of $\lambda$. The resulting rdf curves as well as the rdf residuals are shown in Figure 5. These results show that there is an optimal set of parameters in the space $(\varepsilon, \sigma, \lambda)$ that simultaneously reproduces the enthalpy of vaporization and density of liquid water and minimizes $\mathrm{rdf}_{\text {res }}$. These parameters are $\sigma=2.3826 \AA, \varepsilon=6.1504 \mathrm{kcal} / \mathrm{mol}$, and $\lambda=23.743$. We denote the model with these parameters $\mathrm{mW}_{\mathrm{UQ}}$. The predicted $\lambda$ that minimizes the $\mathrm{rdf}_{\text {res }}$ is only $2.6 \%$ higher than the value in the standard $\mathrm{mW}$ model 23.15. A simulation using these 


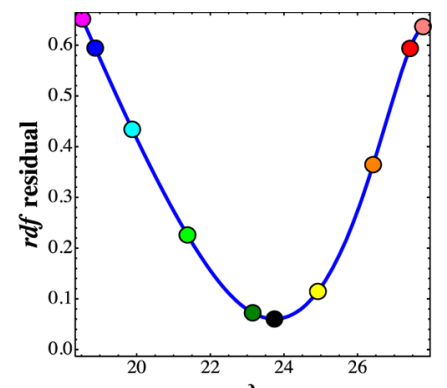

$\lambda$

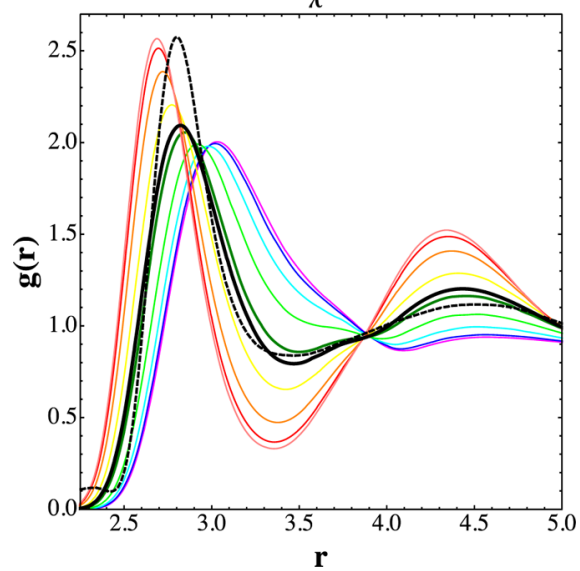

Figure 5. Residual of the radial distribution function $g(r)$ as a function of $\lambda$ (upper panel). For each value of $\lambda$ on this curve, the corresponding values of $\varepsilon$ and $\sigma$ are those that simultaneously match experimental enthalpy of vaporization and density as determined using the response surfaces in the $(\varepsilon, \sigma, \lambda)$ parameter space. The radial distribution functions $g(r)$ for various values of $\lambda$ (lower panel). The rdf curves on the lower panel are color-coded to the points on the upper panel. The dashed line indicates the rdf determined from experimental measurements in ref 58 .

optimized parameters results in $\Delta H_{\text {vap }}$ of $10.52 \mathrm{kcal} / \mathrm{mol}$ and a density of $0.997 \mathrm{~g} / \mathrm{mL}$, matching the experimental results exactly. The $\mathrm{rdf}_{\text {res }}$ calculated from the simulation is 0.058 , compared to the value predicted from interpolation function, 0.060 . The results of various output properties for the $\mathrm{mW}$ and $\mathrm{mW}_{\mathrm{UQ}}$ as well as the experiment are presented in Table 1.

The original parametrization of $\mathrm{mW}$ did not consider the $\mathrm{rdf}$ or adf in the parametrization and matched $T_{\mathrm{m}}$ to $274 \mathrm{~K}$. The $T_{\mathrm{m}}$ for $\mathrm{mW}_{\mathrm{UQ}}$ model is $287 \mathrm{~K}, 5 \%$ above the experimental value of $273.15 \mathrm{~K}$. Although the melting temperature of ice for $\mathrm{mW}_{\mathrm{UQ}}$ is

Table 1. Output Properties for $\mathrm{mW}_{\mathrm{UQ}}$ Compared to $\mathrm{mW}$ and Experiment

\begin{tabular}{llll} 
& \multicolumn{1}{c}{$\exp$} & $\mathrm{mW}_{\mathrm{UQ}}{ }^{a}$ & $\mathrm{~mW}$ \\
$T_{\mathrm{m}}(\mathrm{K})$ & 273.15 & 287 & 274 \\
$T_{\mathrm{MD}}(\mathrm{K})$ & 277 & 264 & 250 \\
$\left.\gamma_{\mathrm{lv}}(\mathrm{mJ} \mathrm{m})^{-2}\right)$ & 71.6 & 63.8 & 66.0 \\
$t_{90-10}(\AA)$ & & 2.8 & 3.3 \\
$\rho\left(\mathrm{g} \mathrm{cm}^{-3}\right)$ & 0.997 & 0.997 & 0.997 \\
$\Delta H_{\mathrm{vap}}\left(\mathrm{kcal} \mathrm{mol}^{-1}\right)$ & 10.52 & 10.52 & 10.65 \\
$\Delta H_{\mathrm{m}}\left(\mathrm{kcal} \mathrm{mol}^{-1}\right)$ & 1.436 & 1.32 & 1.26 \\
$\Delta S_{\mathrm{m}}\left(\mathrm{cal} \mathrm{mol}^{-1} \mathrm{~K}^{-1}\right)$ & 5.257 & 4.60 & 4.60 \\
$D\left(10^{-5} \mathrm{~cm}^{2} \mathrm{~s}^{-1}\right)$ & 2.3 & 5.9 & 6.5 \\
$\operatorname{rdf}_{\mathrm{res}}\left(\AA^{2}\right)$ & 0 & 0.06 & 0.07
\end{tabular}

${ }^{a} \mathrm{~mW}_{\mathrm{UQ}}$ parameters are $\sigma=2.3826 \AA, \varepsilon=6.1504 \mathrm{kcal} / \mathrm{mol}$, and $\lambda=$ 23.743 . not as accurate as for the original $\mathrm{mW}$ model, it is still more accurate than all popular atomistic water models except TIP4P/ ice, which was parametrized to reproduce this property. ${ }^{62}$ The $T_{\mathrm{MD}}$ for $\mathrm{mW}_{\mathrm{UQ}}$ is $23 \mathrm{~K}$ below the $T_{\mathrm{m}}$, similar to the $\mathrm{mW}$ model and $5 \%$ lower than the experimental value of $277 \mathrm{~K}$. The lack of hydrogen atoms in this family of coarse-grained models results in the loss of all rotational contributions to the entropy. This precludes the possibility of simultaneously reproducing $T_{\mathrm{m}}$ and $\Delta H_{\mathrm{m}}$. The original $\mathrm{mW}$ model reproduces the melting temperature because it reproduces the ratio of $\Delta H_{\mathrm{m}} / \Delta S_{\mathrm{m}}=$ $T_{\mathrm{m}} . \Delta H_{\mathrm{m}}$ and $\Delta S_{\mathrm{m}}$ in $\mathrm{mW}$, however, are both $12 \%$ lower than experiment. ${ }^{42}$ We note that previous investigations using the Stillinger-Weber model indicate that $\Delta S_{\mathrm{m}}$ of the tetrahedral crystal is quite insensitive to $\lambda^{69}$ For the $\mathrm{mW}_{\mathrm{UQ}}$ model parameters that optimize the structure, density and enthalpy of vaporization of the liquid, the improvement in $\Delta H_{\mathrm{m}}$ is counterbalanced by an overestimation of $T_{\mathrm{m}}$ because $\Delta S_{\mathrm{m}}$ remains unchanged.

The self-diffusion coefficient $D$ for $\mathrm{mW}_{\mathrm{UQ}}$ is $5.9 \times 10^{-5} \mathrm{~cm}^{2}$ $\mathrm{s}^{-1}$, slightly improved over the value for $\mathrm{mW}\left(6.5 \times 10^{-5} \mathrm{~cm}^{2}\right.$ $\left.\mathrm{s}^{-1}\right)$, but still 2.6 times higher than the experimental value. The monatomic nature of this coarse-grained model precludes the reproduction of experimental values of the diffusion constant while also reproducing the structure and thermodynamics. The loss of the hydrogen atoms results in fewer degrees of freedom and a smoother potential energy surface. Therefore, dynamic properties such as diffusion, which depend on the magnitude of the barriers for rearrangement of hydrogen bonds, are not reproduced by any of the coarse-grained water models that represent water as a single particle or map several water molecules into a single bead.

The results of this section illustrate that UQ can be used as a method to parametrize coarse-grained models using an arbitrary number of properties of interest, and to assess the accuracy, representability and transferability of the model with respect to these and other properties. These same methods would also work to parametrize an atomistic model using a "top-down" approach. The selection of experimental properties to match in the parametrization determines the overall quality of the model. ${ }^{62}$ The $\mathrm{mW}_{\mathrm{UQ}}$ model with $\sigma=2.3826 \AA$, $\varepsilon=6.1504$ $\mathrm{kcal} / \mathrm{mol}$, and $\lambda=23.743$ is very close in parameter space and performance to the original $\mathrm{mW}$ model $(\sigma=2.3925 \AA, \varepsilon=$ $6.189 \mathrm{kcal} / \mathrm{mol}$, and $\lambda=23.15)$. The $\mathrm{mW}_{\mathrm{UQ}}$ parametrization improves slightly the accuracy on rdf, $T_{\mathrm{MD}}, \Delta H_{\mathrm{vap}}$, and $\Delta H_{\mathrm{m}}$ at the expense of a small loss in accuracy in $T_{\mathrm{m}}$ and the liquidvapor surface tension $\gamma_{\mathrm{lv}}$. The overall global agreement of the two parametrizations of the monatomic water model, as measured by the sum of the deviations with respect to all experimental properties in Table 1 , is comparable. In the following section, we use UQ methods not merely to parametrize a model, but to better understand the effect of the length of the interaction potential on the accuracy of the model and its representability.

\section{HOW SHORT IS TOO SHORT FOR WATER INTERACTIONS?}

The length of the interaction between water molecules in the $\mathrm{mW}$ model is controlled by the parameters $\sigma$ and $a$. Their product determines the distance at which the two-body and three-body terms of the interaction potential go to zero (upper and middle panels of Figure 6). The parameter $\gamma$ scales the magnitude of the three-body potential energy term (lower panel of Figure 6). To investigate the role of the length of the 

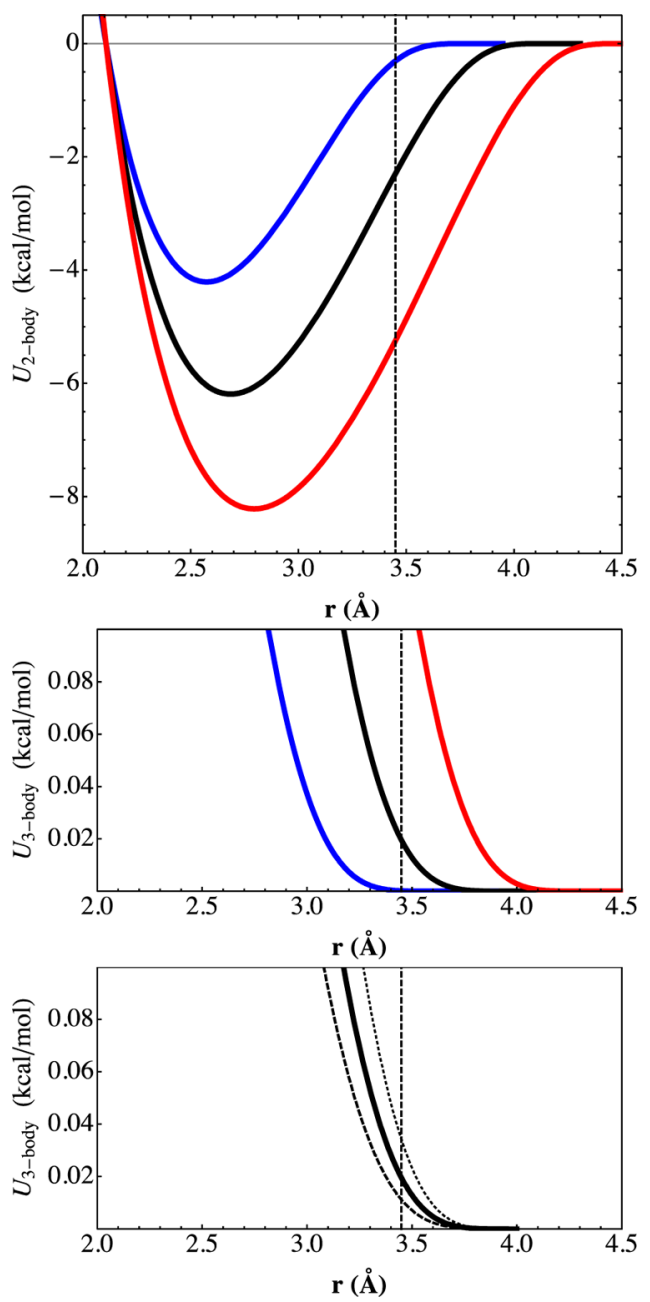

Figure 6. Two-body interaction potential as a function of $a$ (upper panel). Three-body interaction potential as a function of $a$ (middle panel). Three-body interaction potential as a function of $\gamma$ (lower panel). The product $\sigma a$ determines the cutoff of the interaction potential. The solid black line represents the potential using the standard $\mathrm{mW}$ parameters $a=1.8(\sigma a=4.31 \AA)$ and $\gamma=1.2$. A blue line indicates $a=1.65(\sigma a=3.95 \AA)$, and a red line indicates $a=1.95(\sigma a$ $=4.67 \AA$ ). The dashed black line indicates $\gamma=1.3$, and the dotted black line indicates $\gamma=1.1$. To visualize the three-body term of eq 1 , the constraints $r_{i j}=r_{i k}$ and $\theta_{i j k}=90^{\circ}$ are imposed. All other parameters have the same value as in the standard $\mathrm{mW}$ model.

interaction potential cutoff on the output properties, we constructed the response surfaces as a function of the $a$ and $\gamma$ parameters while maintaining all other parameters at their values in the standard $\mathrm{mW}$ model. Figure 7 shows the density as a function of $\sigma a$ and $\gamma$. The surfaces as a function of the length scale of the potential $\sigma a$ and the scale of the three-body potential $\gamma$ display a common behavior: the properties change smoothly except for the region of $\sigma a$ shorter than $3.95 \AA$ and except when $\gamma$ deviates from 1.2, for which the properties differ radically from those of water. This is associated with the inability to hold together the structure of water in the first shell, which extends from about 2.5 to $3.5 \AA$, when the range of the interactions is shorter than the first peak of the radial distribution function. Note that due to the form of the potential, even when the cutoff $\sigma a$ is longer than $3.5 \AA$, e.g., for $\sigma a=3.95 \AA$ in Figure 6, the interactions within the first shell are severely diminished.

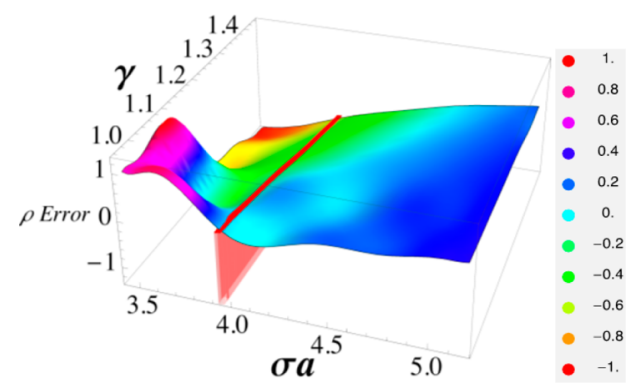

Figure 7. Relative error of the density of liquid water with respect to experiment as a function of parameters $\gamma$ and $\sigma a(\sigma=2.3925 \AA)$. The interaction potential cutoff distance is determined by the product $\sigma a$. Below $\sigma a=3.95 \AA$ (red line), the first shell neighbors do not sufficiently interact with each other, resulting in a low density state for low $\gamma$ and high density state for high $\gamma$. Values of $\gamma$ smaller than 1.2 increase the value of the three-body interaction potential and promote the separation of particles. Values of $\gamma$ larger than 1.2 decrease the three-body repulsion and promote the condensation of particles.

We find that the interaction length that reproduces the experimental values for $\rho$ and $\Delta H_{\text {vap }}$ of water is limited to a small region of parameter space centered around $a=1.8(\sigma a=$ $4.31 \AA$ A). Figure 8 displays the loci of optimum $\Delta H_{\text {vap }}$ and $\rho$ along with the contour plots of $\operatorname{rdf}_{\text {res }}$ (upper panel) and $\operatorname{adf}_{\text {res }}$ (lower panel) as a function of $a$ and $\gamma$. The family of parametrizations with $a=1.65$ (cutoff $\sigma a=3.95 \AA$ ) either reproduces the density at the expense of the enthalpy of vaporization or vice versa. All parametrizations with this shorter cutoff sacrifice the accuracy of the rdf and also deviate significantly in the prediction of most other properties, as shown in Table 2 . Note the wild variations of the liquid vapor surface tension, $\gamma_{\mathrm{lv}}$, and width of the water-vapor interface, $t_{90-10}$, on changing $\gamma$ for the potentials with $\sigma a=3.95 \AA$. The decrease in tetrahedral interactions on increasing $\gamma$ and the decrease in length scale of the potential on lowering $a$ result in a destabilization of the tetrahedral ice crystal and the disappearance of the density anomaly. These very short potentials fail at producing, even qualitatively, the characteristic properties of water.

The parameter $\gamma$ is able to compensate for the tetrahedral parameter $\lambda$ in the three-body potential function. As $\gamma$ decreases, the energetic penalty for nontetrahedral three-body configurations increases, mimicking the effect of increasing $\lambda$. The parameter $a$ is also able to partially compensate for changes in $\lambda$. For example, in the three-body potential reducing $\sigma a$ from 4.31 to $3.95 \AA$ ( $8 \%$ reduction) affects the magnitude of the potential (at $r_{i j}=r_{i k}=\sigma$ and $\theta_{i j k}=90^{\circ}$ ) in a way similar to reducing $\lambda$ by $50 \%$. At the same time, reducing $a$ decreases the cutoff distance of the two-body and three-body terms. Can an increase in tetrahedral order (either increase $\lambda$ or decrease $\gamma$ ) compensate for the effect of a very short potential? Figure 6 shows that reducing a diminishes the control of the tetrahedrality of the model (because of a shorter cutoff), leaving $\lambda$ or $\gamma$ less able to compensate for this loss. In the evaluation above of the surfaces for $(a, \gamma)$, all the other parameters were fixed to their values in the standard $\mathrm{mW}$ model. However, because some of the parameters in this model can compensate for each other to potentially improve the parametrization for different interaction cutoffs, there may be a set of parameters for other values of $a$ that result in more accurate parametrizations of water. In what follows we use UQ 

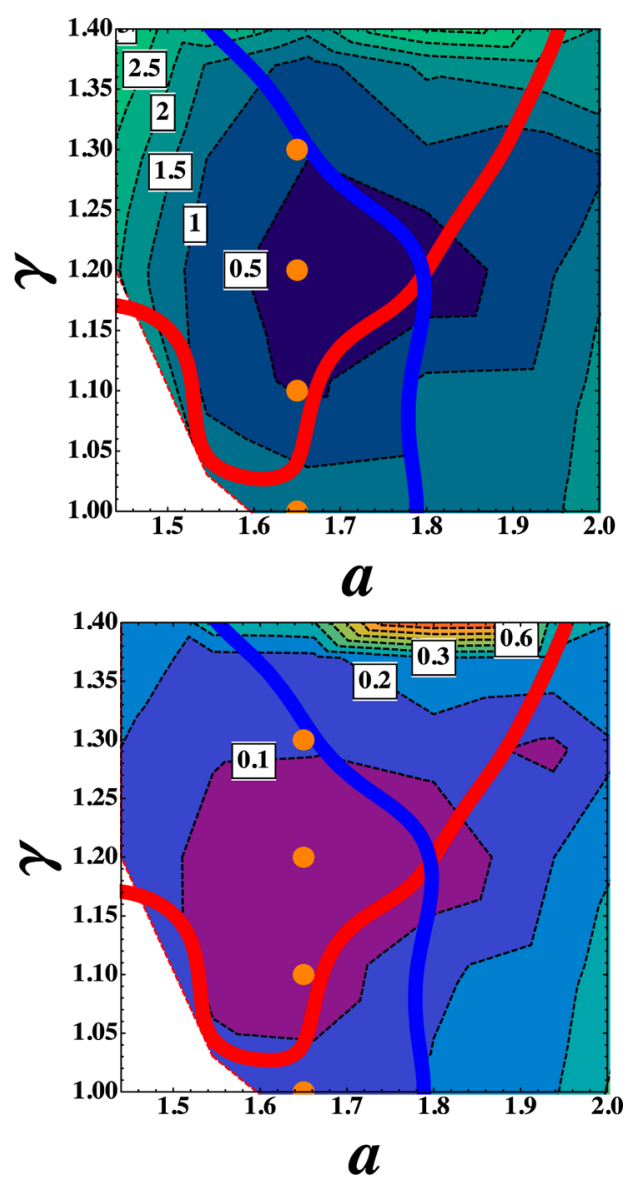

Figure 8. Simultaneous optimization of the enthalpy of vaporization, density, and structure in the parameter space of $\gamma$ and $a$. Regions in parameter space that match the experimental density (blue line) and enthalpy of vaporization (red line) are overlaid on the rdf residual (upper panel) and the adf residual (lower panel). The orange circles represent the points in parameter space for the simulations with $a=$ $1.65(\sigma a=3.95 \AA)$ listed in Table 2 . The values of the adf residual have been scaled up by 1000 to aid in the comparison to the rdf.

to find the best set of parameters as a function of the cutoff length $a$.

To investigate the role of the interaction length on the physical properties of short-ranged, anisotropic three-body water models, we evaluated the accuracy of the model as a function of the cutoff distance $a$. To allow the model parameters to compensate for each other to produce the most accurate representations of water for each cutoff distance, we constructed a three-dimensional response surface as a function of the parameters $\sigma, a$, and $\lambda$. For each value of $a$, we found the combination of parameters that minimized $\mathrm{rdf}_{\text {res }}$. Because $\lambda$ and $\gamma$ are able to compensate for each other, we fixed $\gamma$ at 1.2. We scaled the strength of interaction potential $\varepsilon$ so that each of the parameter sets had the same depth of the attractive potential well $(6.189 \mathrm{kcal} / \mathrm{mol})$. The cutoff distance of the interaction potential is given by the product of $\sigma$ and $a$. The resulting two- and three-body interaction potentials represent the potential functions that have the combination of parameters $\sigma$ and $\lambda$ that minimize $\operatorname{rdf}_{\text {res }}$ for each value of $a$ and are shown in Figure 9. Table 3 summarizes the output properties as a function of the length of the interaction potential.
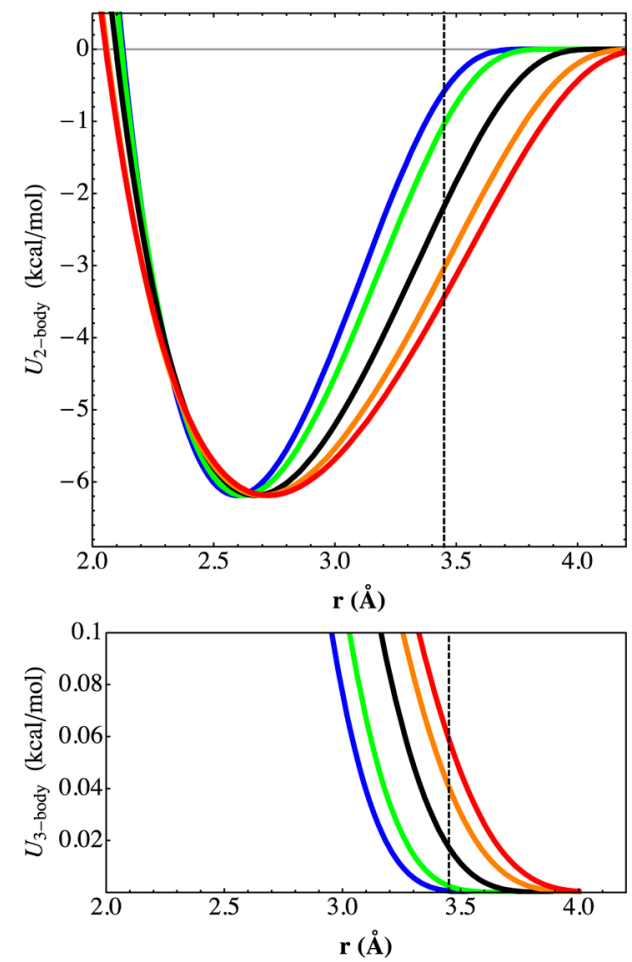

Figure 9. Interaction potential curves for the two-body interactions (upper panel) and three-body interactions (lower panel) with increasing interaction cutoff length $\sigma a$. The parameters $\sigma$ and $\lambda$ for each curve are the optimized values taken from the response surface for the parameter space $(a, \sigma, \lambda)$ as a function of $a$. The parameter $\varepsilon$ is scaled so that each of the parameter sets had the same depth of the attractive potential well $(6.189 \mathrm{kcal} / \mathrm{mol})$. These parameters are listed in Table 3. To visualize the three-body term of eq 1 , the constraints $r_{i j}$ $=r_{i k}$ and $\theta_{i j k}=90^{\circ}$ are imposed. All other parameters have the same value as in the standard $\mathrm{mW}$ model. The dashed line indicates the first minimum in the radial distribution function $3.45 \AA$ from ref 58 . The cutoff lengths defined by $\sigma a$ are $3.98,4.08,4.29,4.46$, and $4.55 \AA$ for the blue, green, black, orange and red curves, respectively.

Table 2. Effect of $\gamma$ on the Properties of the Model for $a \sigma=3.95 \AA$

\begin{tabular}{|c|c|c|c|c|c|c|}
\hline & $\exp$ & $\mathrm{mW}$ & $\gamma=1.0$ & $\gamma=1.1$ & $\gamma=1.2$ & $\gamma=1.3$ \\
\hline$T_{\mathrm{m}}(\mathrm{K})$ & 273.15 & 274 & 321 & 240 & $\mathrm{NA}^{a}$ & $\mathrm{NA}^{a}$ \\
\hline$T_{\mathrm{MD}}(\mathrm{K})$ & 277 & 250 & 209 & 218 & $\mathrm{NA}^{b}$ & $\mathrm{NA}^{b}$ \\
\hline$\gamma_{\mathrm{lv}}\left(\mathrm{mJ} \mathrm{m}^{-2}\right)$ & 71.6 & 66.0 & 16.1 & 28.9 & 59.4 & 111 \\
\hline$t_{90-10}(\AA)$ & & 3.3 & 8.9 & 5.5 & 4.7 & 2.8 \\
\hline$\rho\left(\mathrm{g} \mathrm{cm}^{-3}\right)$ & 0.997 & 0.997 & 0.922 & 1.06 & 1.31 & 1.53 \\
\hline$\Delta H_{\text {vap }}\left(\mathrm{kcal} \mathrm{mol}^{-1}\right)$ & 10.52 & 10.65 & 6.40 & 6.64 & 7.98 & 10.25 \\
\hline$D\left(10^{-5} \mathrm{~cm}^{2} \mathrm{~s}^{-1}\right)$ & 2.3 & 6.5 & 2.2 & 8.8 & 12.0 & 4.2 \\
\hline $\operatorname{rdf}_{\mathrm{res}}\left(\AA^{2}\right)$ & 0 & 0.07 & 1.2 & 0.4 & 0.2 & 0.5 \\
\hline
\end{tabular}

${ }^{a}$ The crystal was not stable. ${ }^{b}$ The liquid vitrifies without displaying a density maximum. 
Table 3. Properties of Water Models as a Function of Interaction Length

\begin{tabular}{|c|c|c|c|c|c|c|c|}
\hline & \multirow[b]{2}{*}{$\exp$} & \multicolumn{6}{|c|}{$\sigma a(\AA)$} \\
\hline & & $4.31(\mathrm{~mW})$ & 3.98 & 4.08 & 4.29 & 4.46 & 4.55 \\
\hline$a$ & & 1.8 & 1.65 & 1.6939 & 1.8 & 1.9061 & 1.95 \\
\hline$\sigma(\AA)$ & & 2.3925 & 2.4127 & 2.4067 & 2.3807 & 2.3388 & 2.3332 \\
\hline$\lambda$ & & 23.15 & 27.78 & 27.78 & 23.57 & 19.06 & 18.52 \\
\hline$\varepsilon\left(\mathrm{kcal} \mathrm{mol}^{-1}\right)$ & & 6.189 & 9.102 & 8.019 & 6.189 & 5.025 & 4.662 \\
\hline$T_{\mathrm{m}}(\mathrm{K})$ & 273.15 & 274 & 272 & 263 & 286 & 262 & 282 \\
\hline$T_{\mathrm{MD}}(\mathrm{K})$ & 277 & 250 & $\mathrm{NA}^{a}$ & 235 & 256 & 227 & 212 \\
\hline$\gamma_{\mathrm{lv}}\left(\mathrm{mJ} \mathrm{m} \mathrm{m}^{-2}\right)$ & 71.6 & 66.0 & 82.7 & 70.9 & 64.4 & 66.5 & 62.1 \\
\hline$t_{90-10}(\AA)$ & & 3.3 & 2.8 & 3.1 & 3.1 & 3.1 & 3.6 \\
\hline$\rho\left(\mathrm{g} \mathrm{cm}^{-3}\right)$ & 0.997 & 0.997 & 1.24 & 1.13 & 1.00 & 0.948 & 0.892 \\
\hline$\Delta H_{\text {vap }}\left(\mathrm{kcal} \mathrm{mol}^{-1}\right)$ & 10.52 & 10.65 & 11.0 & 10.7 & 10.6 & 10.9 & 10.7 \\
\hline$D\left(10^{-5} \mathrm{~cm}^{2} \mathrm{~s}^{-1}\right)$ & 2.3 & 6.5 & 7.7 & 6.7 & 6.1 & 7.4 & 6.5 \\
\hline $\operatorname{rdf}_{\text {res }}\left(\AA^{2}\right)$ & 0 & 0.07 & 0.11 & 0.07 & 0.06 & 0.14 & 0.17 \\
\hline
\end{tabular}

The cutoff length that best reproduces the structure and energetics of water is $4.29 \AA$, essentially indistinguishable from the $4.31 \AA$ cutoff of the standard $\mathrm{mW}$ model. The parameters that minimize the $\mathrm{rdf}_{\mathrm{res}}$ do not reproduce the experimental density exactly, but they are still quite close (within 3\%). Improving the density by rescaling $\sigma$ would also increase $\mathrm{rdf}_{\text {res }}$. The agreement with $\Delta H_{\text {vap }}$ can be improved by rescaling $\varepsilon$, but the best simultaneous agreement with structure and energetic properties is centered at $a=1.8$. Not surprisingly, the accuracy of the model decreases as the cutoff becomes shorter. Interestingly, interaction potentials extending longer than $4.29 \AA$ do not improve the accuracy of the model but actually diminish it. Using the effective potential to recreate the tetrahedral structure of water becomes unphysical when the interaction potential is integrated much beyond the first solvation shell. These results indicate that interactions with the first neighbor shell have to be fully accounted for, and that subtle changes in the interaction with the first neighbor shell greatly affect the physics of water. It should be noted that the models used for the preceding analysis use the same cutoff distance for the two- and three-body terms. It is possible to add another degree of freedom by creating separate cutoff distances for the two- and three-body terms will yield a different optimum length scale. In coarse-grained parametrizations determined by force matching and relative entropy minimization, the cutoff lengths are different for the two- and three-body interactions, but the agreement with the target atomistic SPC/E model (and experimental) $\Delta H_{\text {vap }}$ is poor despite the accuracy with respect to the rdf and adf of the atomistic model, which are not exactly the same as in the experiment. ${ }^{3,14}$ Figure 8 shows that as the interaction length of the water model decreases, the accuracy of the enthalpy and density suffer and adjusting parameters to fix one diminishes the other, although the structure is still well reproduced, suggesting that this limitation of short models is beyond the nature of the target model used for the parametrization. The inability of very short ranged anisotropic monatomic water models to reproduce simultaneously the density and enthalpy of water is not limited to SW potentials that, like $\mathrm{mW}$ and the variants investigated in this work, use the same cutoff for the two- and three-body interactions but is manifest also in SW monatomic water models parametrized using relative entropy minimization allowing for distinct cutoffs of the two- and three-body interactions, ${ }^{14}$ as well as in monatomic water models optimized with force matching that not only employ different cutoffs for the three-body and two-body interactions but also use very flexible spline functions to represent the latter. ${ }^{3,13}$ Therefore, we expect the representability issues of very short-models to be independent of the specific form of the interaction potential.

The structure and thermodynamics of water are simultaneously and quite accurately reproduced for the interaction length scale of the standard $\mathrm{mW}$ model. Even though the radial and angular distribution functions of water were not used for the original $\mathrm{mW}$ parametrization, the model does an excellent job at reproducing them as well as the density and energetics of liquid water leaving surprisingly little room for improvement within this paradigm. Also surprising is that the choice for the interaction cutoff length of $a=1.8$ in the original Stillinger and Weber silicon potential is the locus for the optimal interaction length for the water model.

\section{CONCLUSIONS}

We performed uncertainty quantification by generalized polynomial chaos using Lagrange interpolation with Chebyshev nodes to assess the accuracy and representability of a family of coarse-grained water models as a function of the model input parameters. The hierarchical nature of the Lagrange interpolation allows for more efficient sampling than other UQ methods. The nested hierarchy and ability to evaluate the sensitivity of the model to input parameters can help determine where to invest computational resources during the parametrization of model potentials. Simulation models are not capable of simultaneously reproducing all properties of interest, but UQ allows for a more comprehensive understanding of which properties suffer at the expense of others. UQ can be used to efficiently evaluate the transferability of a model over a wide range of state points with fewer computational resources than in traditional approaches, as we illustrated for the temperature dependence of the density of liquid water. These methods should be viewed as part of a holistic approach that can be coupled with other force-field parametrization methods such as force-matching and relative entropy minimization to develop more accurate models and a deeper understanding of molecular systems.

The overall quality of the resulting coarse-grained model depends on which output properties of water are used as targets. Parameters that prioritize the structure of liquid water are different from those that privilege the accurate reproduction of the melting temperature of hexagonal ice. Nevertheless, we found that the parametrization that optimizes density, enthalpy 
of vaporization and $\mathrm{rdf}$ is very close to the standard $\mathrm{mW}$ parametrization based on density, enthalpy of vaporization and melting temperature of ice. For the family of coarse-grained models of this study, the diffusivity is never accurately reproduced simultaneously with the energetics and structure. This represents an intrinsic limitation of the model associated to the reduction in the entropy due to the loss of degrees of freedom and cannot be alleviated by reparametrization.

The cutoff length for the potential determines the ability of the monatomic anisotropic model to simultaneously reproduce the structure and energetics of water. The structure and thermodynamics of water can be simultaneously reproduced with a monatomic model that uses short two- and three-body interaction potentials that act over a distance slightly longer than the first solvation shell of the water-water radial distribution function. The interaction length that satisfies these two criteria is limited to a small region of parameter space centered at the cutoff distance $4.3 \AA$ for the functional form of the Stillinger-Weber potential. Models with cutoffs that are shorter and longer than this optimal distance do not reproduce the structure of water as measured in experiment, and attempts to compensate by changing other parameters degrades the accuracy of the energetics. We conclude that the length scale relevant for interactions in water is, as predicted 80 years ago by Bernal and Fowler, barely beyond the first neighbor shell.

\section{AUTHOR INFORMATION}

\section{Corresponding Author}

*V. Molinero: e-mail, Valeria.Molinero@utah.edu.

\section{Notes}

The authors declare no competing financial interest.

\section{ACKNOWLEDGMENTS}

The authors gratefully acknowledge Hadi Meidani for helpful discussions and the Center of High Performance Computing at the University of Utah for technical support and allocation of computing time. This research was sponsored by the Army Research Laboratory under Cooperative Agreement Number W911NF-12-2-0023. The views and conclusions contained in this document are those of the authors and should not be interpreted as representing the official policies, either expressed or implied, of the Army Research Laboratory or the U.S. Government. The U.S. Government is authorized to reproduce and distribute reprints for Government purposes notwithstanding any copyright notation herein.

\section{REFERENCES}

(1) Noid, W. G. Perspective: Coarse-Grained Models For Biomolecular Systems. J. Chem. Phys. 2013, 139 (9), 090901.

(2) Izvekov, S.; Voth, G. A. Multiscale Coarse Graining Of LiquidState Systems. J. Chem. Phys. 2005, 123 (13), 134105.

(3) Larini, L.; Lu, L.; Voth, G. A. The Multiscale Coarse-Graining Method. VI. Implementation of Three-Body Coarse-Grained Potentials. J. Chem. Phys. 2010, 132, 164107.

(4) Shell, M. S. The Relative Entropy Is Fundamental to Multiscale and Inverse Thermodynamic Problems. J. Chem. Phys. 2008, 129, 144108.

(5) Chaimovich, A.; Shell, M. S. Relative Entropy as a Universal Metric For Multiscale Errors. Phys. Rev. E 2010, 81, 060104.

(6) Carmichael, S. P.; Shell, M. S. A New Multiscale Algorithm and its Application to Coarse-Grained Peptide Models for Self-Assembly. J. Phys. Chem. B 2012, 116, 8383-8393.
(7) Reith, D.; Pütz, M.; Müller-Plathe, F. Deriving Effective Mesoscale Potentials From Atomistic Simulations. J. Comput. Chem. 2003, 24, 1624-1636.

(8) Lyubartsev, A. P.; Laaksonen, A. Calculation of Effective Interaction Potentials From Radial Distribution Functions: A Reverse Monte Carlo Approach. Phys. Rev. E 1995, 52, 3730-3737.

(9) Head-Gordon, T.; Stillinger, F. H. An Orientational Perturbation Theory for Pure Liquid Water. J. Chem. Phys. 1993, 98, 3313-3327.

(10) Garde, S.; Ashbaugh, H. S. Temperature Dependence of Hydrophobic Hydration and Entropy Convergence in an Isotropic Model of Water. J. Chem. Phys. 2001, 115, 977-982.

(11) Noid, W. G.; Chu, J.-W.; Ayton, G. S.; Krishna, V.; Izvekov, S.; Voth, G. A.; Das, A.; Andersen, H. C. The Multiscale Coarse-Graining Method. I. A Rigorous Bridge between Atomistic and Coarse-Grained Models. J. Chem. Phys. 2008, 128, 244114.

(12) Das, A.; Andersen, H. C. The Multiscale Coarse-Graining Method. IX. A General Method for Construction of Three Body Coarse-Grained Force Fields. J. Chem. Phys. 2012, 136, 194114.

(13) Larini, L.; Shea, J.-E. Double Resolution Model for Studying TMAO/Water Effective Interactions. J. Phys. Chem. B 2013, 117, 13268-13277.

(14) Lu, J.; Qiu, Y.; Baron, R.; Molinero, V. Manuscript to be submitted for publication.

(15) Chernatynskiy, A.; Phillpot, S. R.; Lesar, R. Uncertainty Quantification in Multiscale Simulation of Materials: A Prospective. Annu. Rev. Mater. Res. 2013, 43, 157-182.

(16) Fox, B. L. Strategies for Quasi-Monte Carlo; Kluwer Academic: Boston, 1999.

(17) Loh, W.-L. On Latin Hypercube Sampling. Ann. Stat. 1996, 24, 2058-2080.

(18) Stein, M. Large Sample Properties of Simulations Using Latin Hypercube Sampling. Technometrics 1987, 29, 143-151.

(19) Niederreiter, H. H. P.; Larcher, G.; Zinterhof, P. Monte Carlo and Quasi-Monte Carlo Methods; Springer-Verlag: Berlin, 1998; Vol. 127, p 448

(20) Ghanem, R. G.; Spanos, P. D., Stochastic Finite Elements: A Spectral Approach; Dover Publications, 2003.

(21) Xiu, D.; Karniadakis, G. E. Modeling Uncertainty In Flow Simulations via Generalized Polynomial Chaos. J. Comput. Phys. 2003, 187, 137-167.

(22) Maître, O. P. L.; Knio, O. M. Spectral Methods For Uncertainty Quantification: With Applications to Computational Fluid Dynamics; Springer: Berlin, 2010.

(23) Sudret, B. Global Sensitivity Analysis Using Polynomial Chaos Expansions. Reliability Engineering \& System Safety 2008, 93, 964-979.

(24) Xiu, D.; Hesthaven, J. High-Order Collocation Methods for Differential Equations with Random Inputs. SIAM Journal on Scientific Comput. 2005, 27, 1118-1139.

(25) Ma, X.; Zabaras, N. An Adaptive Hierarchical Sparse Grid Collocation Algorithm for the Solution of Stochastic Differential Equations. J. Comput. Phys. 2009, 228, 3084-3113.

(26) Nobile, F.; Tempone, R.; Webster, C. A Sparse Grid Stochastic Collocation Method for Partial Differential Equations with Random Input Data. SIAM Journal of Numerical Analysis 2008, 46, 2309-2345.

(27) Le MaîTre, O. P.; Knio, O. M.; Najm, H. N.; Ghanem, R. G. Uncertainty Propagation Using Wiener-Haar Expansions. J. Comput. Phys. 2004, 197, 28-57.

(28) Le MaîTre, O. P.; Najm, H. N.; Ghanem, R. G.; Knio, O. M. Multi-Resolution Analysis of Wiener-Type Uncertainty Propagation Schemes. J. Comput. Phys. 2004, 197, 502-531.

(29) Schwab, C.; Todor, R.-A. Sparse Finite Elements for Elliptic Problems with Stochastic Loading. Numerical Mathematics 2003, 95, 707-734.

(30) Wan, X.; Karniadakis, G. Multi-Element Generalized Polynomial Chaos for Arbitrary Probability Measures. SIAM Journal on Scientific Computing 2006, 28, 901-928.

(31) Rizzi, F.; Najm, H. N.; Debusschere, B. J.; Sargsyan, K.; Salloum, M.; Adalsteinsson, H.; Knio, O. M. Uncertainty Quantification in MD 
Simulations. Part I: Forward Propagation. Multiscale Modeling \& Simulation 2012, 10, 1428-1459.

(32) Rizzi, F.; Najm, H. N.; Debusschere, B. J.; Sargsyan, K.; Salloum, M.; Adalsteinsson, H.; Knio, O. M. Uncertainty Quantification In MD Simulations. Part II: Bayesian Inference of Force-Field Parameters. Multiscale Modeling \& Simulation 2012, 10, 1460-1492.

(33) Rizzi, F.; Jones, R. E.; Debusschere, B. J.; Knio, O. M. Uncertainty Quantification in MD Simulations of Concentration Driven Ionic Flow Through a Silica Nanopore. I. Sensitivity to Physical Parameters of the Pore. J. Chem. Phys. 2013, 138, 194104-194119.

(34) Rizzi, F.; Jones, R. E.; Debusschere, B. J.; Knio, O. M. Uncertainty Quantification in MD Simulations of Concentration Driven Ionic Flow Through A Silica Nanopore. II. Uncertain Potential Parameters. J. Chem. Phys. 2013, 138, 194105-194116.

(35) Remsing, R.; Rodgers, J.; Weeks, J. Deconstructing Classical Water Models at Interfaces and in Bulk. J. Stat. Phys. 2011, 145, 313334.

(36) Izvekov, S.; Swanson, J. M. J.; Voth, G. A. Coarse-Graining in Interaction Space: A Systematic Approach for Replacing Long-Range Electrostatics with Short-Range Potentials. J. Phys. Chem. B 2008, 112, 4711-4724.

(37) Hadley, K. R.; Mccabe, C. Coarse-Grained Molecular Models of Water: A Review. Mol. Simul. 2012, 38, 671-681.

(38) Chaimovich, A.; Shell, M. S. Anomalous Waterlike Behavior in Spherically-Symmetric Water Models Optimized with the Relative Entropy. Phys. Chem. Chem. Phys. 2009, 11, 1901-1915.

(39) Johnson, M. E.; Head-Gordon, T.; Louis, A. A. Representability Problems for Coarse-Grained Water Potentials. J. Chem. Phys. 2007, 126, 144509 .

(40) Johnson, M. E.; Head-Gordon, T. Assessing ThermodynamicDynamic Relationships for Water-like Liquids. J. Chem. Phys. 2009, 130, 214510.

(41) Molinero, V.; Goddard, W. A., III. M3B: A Coarse Grain Force Field for Molecular Simulations of Malto-Oligosaccharides and their Water Mixtures. J. Phys. Chem. B 2004, 108, 1414-1427.

(42) Molinero, V.; Moore, E. B. Water Modeled as an Intermediate Element Between Carbon and Silicon. J. Phys. Chem. B 2009, 113, $4008-4016$

(43) Dias, C. L.; Ala-Nissila, T.; Grant, M.; Karttunen, M. ThreeDimensional "Mercedes-Benz" Model for Water. J. Chem. Phys. 2009, 131, 054505.

(44) Darré, L.; Machado, M. A. R.; Dans, P. D.; Herrera, F. E.; Pantano, S. Another Coarse Grain Model for Aqueous Solvation: WAT FOUR? J. Chem. Theory Comput. 2010, 6, 3793-3807.

(45) Wu, Z.; Cui, Q.; Yethiraj, A. A New Coarse-Grained Model for Water: The Importance of Electrostatic Interactions. J. Phys. Chem. B 2010, 114, 10524-10529.

(46) Darré, L.; Machado, M. R.; Pantano, S. Coarse-Grained Models of Water. Wiley Interdiscip. Rev.: Comput. Mol. Sci. 2012, 2, 921-930.

(47) Hujo, W.; Shadrack Jabes, B.; Rana, V.; Chakravarty, C.; Molinero, V. The Rise and Fall of Anomalies in Tetrahedral Liquids. J. Stat. Phys. 2011, 145, 293-312.

(48) Jabes, S. B.; Nayar, D.; Dhabal, D.; Molinero, V.; Chakravarty, C. Water and Other Tetrahedral Liquids: Order, Anomalies and Solvation. J. Phys. Condens. Matter 2012, 24, 284116.

(49) Moore, E. B.; Molinero, V. Structural Transformation in Supercooled Water Controls the Crystallization Rate of Ice. Nature 2011, 479, 506-508.

(50) Moore, E. B.; Molinero, V. Growing Correlation Length in Supercooled Water. J. Chem. Phys. 2009, 130, 244505.

(51) Jacobson, L. C.; Hujo, W.; Molinero, V. Amorphous Precursors in the Nucleation of Clathrate Hydrates. J. Am. Chem. Soc. 2010, 132, 11806-11811.

(52) Baron, R.; Molinero, V. Water-Driven Cavity-Ligand Binding: Comparison of Thermodynamic Signatures from Coarse-Grained and Atomic-Level Simulations. J. Chem. Theory Comput. 2012, 8, 36963704.
(53) Song, B.; Molinero, V. Thermodynamic And Structural Signatures of Water-Driven Methane-Methane Attraction in CoarseGrained mW Water. J. Chem. Phys. 2013, 139, 054511.

(54) Bernal, J. D.; Fowler, R. H. A Theory of Water and Ionic Solution, with Particular Reference to Hydrogen and Hydroxyl Ions. J. Chem. Phys. 1933, 1, 515-548.

(55) Remsing, R. C.; Weeks, J. D. Dissecting Hydrophobic Hydration and Association. J. Phys. Chem. B 2013, 117, 15479-15491.

(56) Stillinger, F. H.; Weber, T. A. Computer Simulation of Local Order in Condensed Phases of Silicon. Phys. Rev. B 1985, 31, 52625271.

(57) Plimpton, S. Fast Parallel Algorithms for Short-Range Molecular Dynamics. J. Comput. Phys. 1995, 117, 1-19.

(58) Skinner, L. B.; Huang, C.; Schlesinger, D.; Pettersson, L. G. M.; Nilsson, A.; Benmore, C. J. Benchmark Oxygen-Oxygen PairDistribution Function of Ambient Water from X-Ray Diffraction Measurements with a Wide Q-Range. J. Chem. Phys. 2013, 138, 074506 .

(59) Strässle, T.; Saitta, A. M.; Godec, Y. L.; Hamel, G.; Klotz, S.; Loveday, J. S.; Nelmes, R. J. Structure of Dense Liquid Water by Neutron Scattering to 6.5 GPa And 670 K. Phys. Rev. Lett. 2006, 96, 067801.

(60) Vega, C.; De Miguel, E. Surface Tension of the Most Popular Models of Water by Using the Test-Area Simulation Method. J. Chem. Phys. 2007, 126, 154707.

(61) Wang, J.; Yoo, S.; Bai, J.; Morris, J. R.; Zeng, X. C. Melting Temperature of Ice Ih Calculated from Coexisting Solid-Liquid Phases. J. Chem. Phys. 2005, 123, 036101.

(62) Vega, C.; Abascal, J. L. F. Simulating Water with Rigid NonPolarizable Models: A General Perspective. Phys. Chem. Chem. Phys. 2011, 13, 19663-19688.

(63) Horn, H. W.; Swope, W. C.; Pitera, J. W.; Madura, J. D.; Dick, T. J.; Hura, G. L.; Head-Gordon, T. Development of an Improved Four-Site Water Model for Biomolecular Simulations: TIP4P-Ew. J. Chem. Phys. 2004, 120, 9665-9678.

(64) Vega, C.; Abascal, J. L. F.; Nezbeda, I. Vapor-Liquid Equilibria From The Triple Point Up To The Critical Point For The New Generation Of Tip4p-Like Models: Tip4p/Ew, Tip4p/2005, And Tip4p/Ice. J. Chem. Phys. 2006, 125 (3), 034503.

(65) Rosenfeld, Y. Relation Between The Transport Coefficients And The Internal Entropy Of Simple Systems. Phys. Rev. A 1977, 15 (6), $2545-2549$.

(66) Errington, J. R.; Truskett, T. M.; Mittal, J. Excess-Entropy-Based Anomalies For A Waterlike Fluid. J. Chem. Phys. 2006, 125 (24), 244502.

(67) Agarwal, M.; Singh, M.; Sharma, R.; Parvez Alam, M.; Chakravarty, C. Relationship Between Structure, Entropy, And Diffusivity In Water And Water-Like Liquids. J. Phys. Chem. B 2010, 114 (20), 6995-7001.

(68) Sengupta, S.; Vasisht, V. V.; Sastry, S. Diffusivity Anomaly In Modified Stillinger-Weber Liquids. J. Chem. Phys. 2014, 140 (4), 044503.

(69) Molinero, V.; Sastry, S.; Angell, C. A. Tuning Of Tetrahedrality In A Silicon Potential Yields A Series Of Monatomic (Metal-Like) Glass Formers Of Very High Fragility. Phys. Rev. Lett. 2006, 97 (7), 075701 . 